\title{
Monofunktionale autonome Transporteinheiten
}

\author{
Dipl.-Ing. Markus Schröppel, Dipl.-Ing. Manuel Weber, \\ Prof. Dr.-Ing. Karl-Heinz Wehking \\ Universität Stuttgart \\ Institut für Fördertechnik und Logistik (Maschinenentwicklung und \\ Materialflussautomatisierung)
}

\begin{abstract}
Ziel eines am Institut für Fördertechnik und Logistik (IFT) laufenden, von der AiF geförderten, Forschungsprojektes ist die Entwicklung eines flexiblen Transportsystems für kleine Transportgüter mit geringem Gewicht. Dazu werden kleine autonome Transporteinheiten (KATE) entwickelt, die sich einerseits selber orientieren können und deren Pose (Fahrzeugposition und Geschwindigkeitsvektor) andererseits z.B. durch ein externes System festgestellt wird. In einem weiteren Projekt des IFT werden kleine autonome Transportfahrzeuge entwickelt, welche den Transport von Paletten ermöglichen. Bei diesen neuartigen Fahrzeugen wurde ein Antriebssystem entwickelt, welches Hub-, Fahr- und Lenkbewegungen kombiniert, um mit denselben Antrieben dem Fahrzeug eine bestimmte Fahrtrichtung vorzugeben, es anzutreiben und die Hubplattform in die Höhe zu bewegen.
\end{abstract}

\section{$1 \quad$ Einleitung}

Bei den fördertechnischen Maschinen werden heutige Unstetigförderer wie Gabelstapler, Verteilwagen, Elektrohängebahnen durch neuartige Kleinfahrzeuge ersetzt werden, die als flexible, selbstorganisierende Transportsysteme aus schwarmfähigen Fahrzeugen für dynamisch konfigurierbare Materialflusssysteme eingesetzt werden. Die angestrebten Merkmale ergeben sich notwendigerweise aus den Anforderungen an zukünftige autonome Unstetigförderersysteme und müssen sich von bestehenden Lösungen aus dem Bereich der fahrerlosen Transportsysteme maßgeblich in technologischer und konstruktiver Hinsicht unterscheiden.
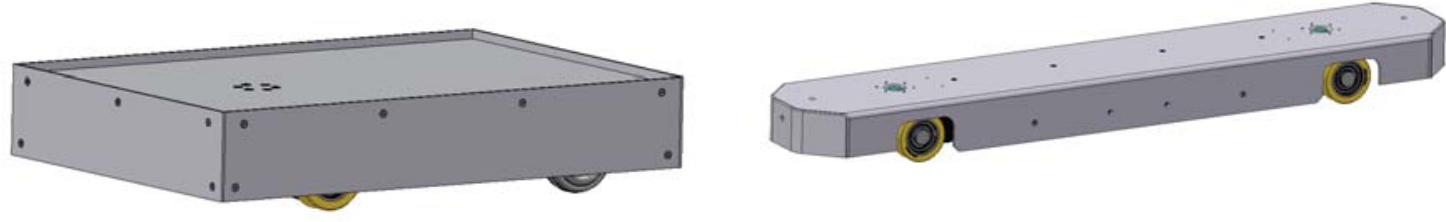

Abbildung 1: Fahrzeug KATE und Transportfahrzeug für Paletten

Dabei sind vor allem monofunktionale Systeme, also Fahrzeuge die z.B. nur eine reine Transportfunktion erfüllen und keine aktiven Lastaufnahmesysteme besitzen, interessant, da diese vergleichsweise einfach und somit kostengünstig ausgeführt (c) 2011 Logistics Journal : Proceedings - ISSN 2192-9084 
werden können und durch die geringere Anzahl an Funktionen auch eine höhere Zuverlässigkeit besitzen. Da in möglichen zukünftigen Systemen sehr viele (z. B. 1000) redundante Fahrzeuge gleichzeitig zum Einsatz kommen werden, ergibt sich als wesentliche Forderung für den wirtschaftlichen Einsatz der Fahrzeuge ein möglichst niedriger Herstellungspreis. Da die Verfügbarkeit des Gesamtsystems nicht mehr durch die Zuverlässigkeit jeder einzelnen Komponente sichergestellt werden muss, soll durch den Einsatz geeigneter Antriebs- und Energieversorgungskonzepte und Werkstoffe für die konstruktive Ausführung der Zielpreis der Fahrzeuge im Vergleich zu heutigen Unstetigförderern einen Quantensprung darstellen.

\section{$2 \quad$ Aufgabenstellung}

Die Fahrzeuge sollen möglichst kompakt und leicht gebaut sein, was den Platz für Energiespeicher stark einschränkt. Dabei sollen zum einen Fahrzeuge entstehen die den Transport von Großladungsträgern wie z.B. Paletten ermöglichen. Zum anderen sollen Fahrzeuge für den Transport von Kleinladungsträgern (KLT) realisiert werden. Für die Navigation von fahrerlosen Transportsystemen gibt es bereits zahlreiche schon in der Praxis eingesetzte Lösungen, wie z.B.:

- induktive Leitdraht- und Transponderführung,

- Laserscannersysteme,

- Kamera,

- Radar,

- Rasternavigation, etc.

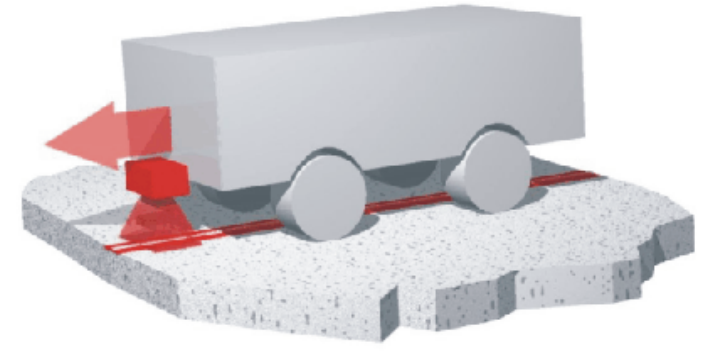

Abbildung 2: Optische Spurführung [Goe11]

Auch zur Datenübertragung zwischen dem Leitrechner und den Fahrzeugen gibt es u.a. folgende Kommunikationslösungen:

- Infrarotübertragung,

- WLAN (IEEE 802.11),

- Bluetooth (IEEE 802.15),

- ZigBee (IEEE 802.15.4, 
- Schmalbandfunk,

- Proprietäres Funksystem im ISM-Band bei 2,4 GHz, etc.

In der ersten Prototypenphase der beiden verschiedenen Fahrzeugkonzepte werden die bereits bekannten und bewährten Systeme zur Navigation und Kommunikation eingesetzt. In dieser Phase liegt das Hauptaugenmerk auf der Umsetzung und Erprobung der Mechanik im eingeschränkten Bauraum und der Energieversorgung für das Gesamtsystem. Parallel wird für die zweite Prototypenphase der Fahrzeuge ein neues System zur Navigation entwickelt, das dann erprobt werden soll.

Der Wirkungsgrad des Antriebs hat einen entscheidenden Einfluss auf den Energieverbrauch, deshalb ist ein besonders effizienter Antriebsstrang erforderlich. Zudem müssen die Komponenten preisgünstig sein, da die Fahrzeuge in großen Stückzahlen eingesetzt werden sollen. Hierbei ist sowohl eine Gleichteileverwendung erforderlich, außerdem kann über ähnliche Komponentenlebensdauer der Wartungsund Reparaturaufwand reduziert werden. Die Energieversorgung muss aufgrund des begrenzt zur Verfügung stehenden Bauraums äußerst kompakt sein. Dabei sollen die Ladezeiten der Energiespeicher möglichst gering sein, um so die Stillstandszeiten zu minimieren.

\section{$3 \quad$ Energiespeicherung}

Zur Speicherung der elektrischen Energie, die zur Versorgung der Fahrzeuge (Antriebsmotoren, Sensoren, Fahrzeugrechner...) benötigt wird soll nach Möglichkeit auf die Verwendung von Akkumulatoren verzichtet werden. Im Rahmen der Projekte am Institut für Fördertechnik und Logistik wird der Einsatz von Kondensatoren als Energiespeicher angestrebt. Diese bieten den Vorteil, dass sie in relativ kurzer Zeit (z.B. $10 \mathrm{~s}$ ) geladen werden können wodurch sich die Verfügbarkeit der Geräte deutlich erhöht. und sie weisen eine Haltbarkeit über eine sehr hohe Anzahl an Ladezyklen (z. B. 500.000 Zyklen) auf [Max11].

Elektrochemische Doppelschichtkondensatoren oder Superkondensatoren (im Englischen electrochemical double layer capacitor - EDLC) bestehen aus 2 mit einem Elektrolyten benetzten Elektroden. Wenn eine Spannung angelegt wird, die unterhalb der Zersetzungsspannung des Elektrolyten liegt, verdichten sich an den beiden Elektroden Ionen mit umgekehrter Polarität. An den Elektroden wird dann eine Zone unbeweglicher Ladungsträger ausgebildet, wobei die Schichtdicke der Zonen nur wenige Moleküllagen beträgt. Doppelschichtkondensatoren werden unter dem Markennamen Goldcaps, Supercaps, Boostcaps oder Ultracaps vertrieben und verfügen über die größte Energiedichte von allen Kondensatoren. Genauso wie bei Elektrolytkondensatoren können Doppelschichtkondensatoren nur gepolt betrieben werden.

Im Gegensatz zu elektrochemischen Energiespeichern, wie beispielsweise Batterien oder Akkus, wird die Energie bei Doppelschichtkondensatoren elektrostatisch gespeichert. Die Supercaps verhalten sich dabei wie eine Reihenschaltung von 2 
Kondensatoren, mit der Ladungsträgerschicht als jeweiliges Dielektrikum. Neben der Schichtdicke der Isolationsschicht hängt die Kapazität eines Supercaps wie bei Elektrolytkondensatoren auch von der Größe der Oberfläche der Elektroden ab. Dabei verfügen Supercaps über sehr hohe Kapazitätsdichten, die ohne weiteres pro Gramm Kondensator 250 Farad betragen können. Die Spannungsfestigkeit eines einzelnen Supercaps liegt bei etwa 2,4-2,7 V. Der davon ableitbare Energieinhalt eines geladenen Kondensators ergibt sich in Abhängigkeit der Kapazität zu $W=1 / 2 \mathrm{CU}^{2}$. Im Vergleich $\mathrm{zu}$ verschiedenen Akkumulatortypen verfügen Doppelschichtkondensatoren über eine sehr hohe spezifische Leistung und einen dementsprechenden sehr geringen Innenwiderstand. Deshalb werden Supercaps eingesetzt, um für kurze Zeiträume hohe Energien, die von einem Verbraucher abgegeben werden können, bzw. verbraucht werden, in Form eines Zwischenspeichers zu puffern.

Wegen der relativ niedrigen Spannungsfestigkeit werden Supercaps üblicherweise in Reihenschaltungen betrieben. Wegen der in der Praxis nicht symmetrischen Ausführung der einzelnen Caps ist die Symmetrierung bei einer Reihenschaltung im praktischen Einsatz eine große Herausforderung und hat einen nennenswerten Einfluss auf den Wirkungsgrad der Energiespeicherung und Abgabe.

Die im Vergleich zu Akkumulatoren um mehrere Zehnerpotenzen höhere Lebensdauer in Ladezyklen von Supercaps prädestiniert sie für Anwendungen mit häufigen Lade- und Entladevorgängen. Eingesetzt werden Supercaps für die Speicherung von Daten in elektronischen Geräten aber zunehmend auch als Energiequelle für mobile Fahrzeuge, die den Einsatz von Rekuperationssystemen vorsehen. Im Vergleich zu Akkumulatoren ist die Ladezeit von Supercaps erheblich geringer. Üblicherweise werden Ladezeiten von deutlich weniger als 1 Minute realisiert. Die Überwachung des Ladezustandes ist ebenfalls im Vergleich zu Akkumulatoren erheblich einfacher, da der Energieinhalt bei bekannter Kapazität nur von der anliegenden Spannung bestimmt wird. Eine Überladung, bzw. Ladestromüberschreitung, ist deshalb kaum möglich. In vielen Anwendungen wird deshalb ein Supercap eingesetzt, um für kurzfristige Lade- oder Entladevorgänge einen hochdynamischen Puffer in Ergänzung zu Standardakkumulatortechnik zu realisieren.

Es ist davon auszugehen, dass in mittelbarer Zukunft, insbesondere bei hybriden Fahrzeugtechnologien, die Bedeutung und der Einsatz von Doppelschichtkondensatoren stark zunehmen wird, um beispielsweise bei Bremsvorgängen kinetische und / oder potentielle Energie nicht in Wärme sondern in nutzbare elektrische Energie umzuwandeln. Beispielsweise werden Doppelschichtkondensatoren auch in Seilbahnkabinen eingesetzt und können wegen der sehr kurzen Ladezeiten bei der Stationsdurchfahrt eines Fahrbetriebsmittels über eine Stromschiene bereits nach einer Durchfahrt vollständig geladen werden. 
Dabei erscheinen für die nähere Zukunft Energiedichten von bis zu 100 Wattstunden $/ \mathrm{kg}$ realistisch. Supercaps stellen deshalb Energiepuffer dar, mit deren Hilfe sich die notwendigen Ladezeiten und durch die zusätzliche Pufferung auch die Lebensdauer von eingesetzten Akkumulatoren erheblich verlängern lässt. Betriebstemperaturen von bis zu $65^{\circ} \mathrm{C}$ stellen kein Problem dar. Das übliche Ladeverfahren von Kondensatoren erfolgt mit einem konstanten Ladestrom, bei Überwachung der Ladespannung auf die höchstzulässige Spannung der Supercaps. Bei Erreichen der Maximalspannung wird der Ladestrom entsprechend der notwendigen Erhaltungsladung des Supercaps reduziert.

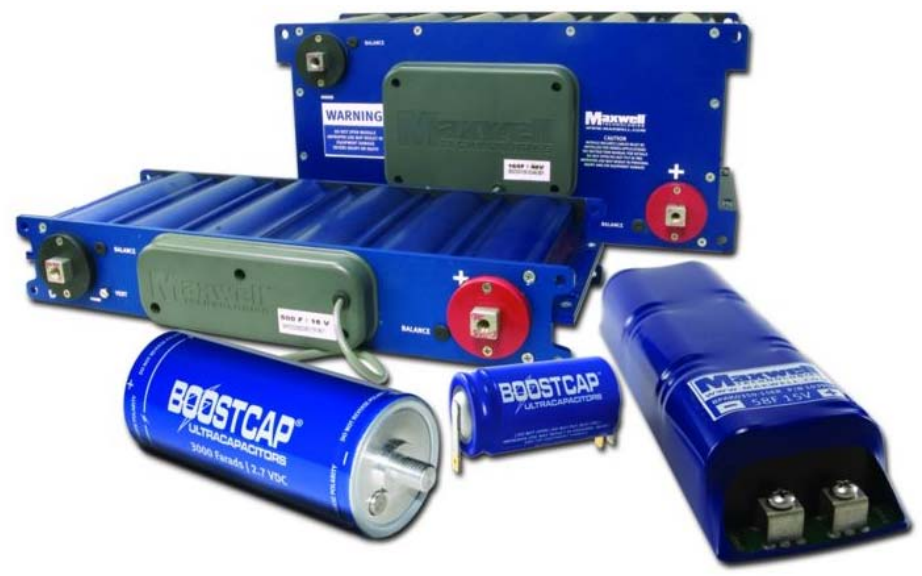

Abbildung 3: Supercap-Pakete mit Symmetrierung über Lastwiderstände [Max11]

Am Markt verfügbare Systeme aus einer Reihenschaltung von Supercaps stellen ein für den Nutzer fertiges Energiespeichersystem mit höheren Spannungen dar. Hierbei kann die Maximalspannung des gesamten Kondensatorpaketes aus dem Produkt der in Reihe geschalteten Supercaps mit der maximalen Einzelspannung ermittelt werden. Die Symmetrierung erfolgt üblicherweise über ohmsche Lastwiderstände (siehe Abbildung 10), was ein einfaches und sicheres Verfahren darstellt aber für den Wirkungsgrad der Energieumsetzung und besonders auch für die Selbstentladung der Akkumulatoren bei längeren Standzeiten nicht die optimale Lösung darstellt.

Bei der im Forschungsprojekt angestrebten Anwendung ist der Wirkungsgrad der Energieumsetzung bei Ladung und Entladung ein entscheidendes Argument und die Selbstentladung spielt insbesondere bei längeren Standzeiten der Fahrzeuge eine große Rolle. Deshalb sollen die für den industriellen Einsatz auch für den Prototyp vorzusehenden Pakete aus Supercaps im Gegensatz zu am Markt verfügbaren Lösungen nicht mit Symmetrierungswiderständen versehen werden, sondern durch eine separate halbleiterbasierte Schaltung auf die Maximalspannung eines jeden einzelnen Kondensators überwacht werden. Um zumindest im Auslieferungszustand eine möglichst optimale Verteilung der Einzelkapazitäten sicherzustellen, wird neben der zur Spannungserhöhung notwendigen Reihenschaltung innerhalb eines jeden Supercap-Paketes auch eine Parallelschaltung von Einzel-caps vorgesehen. Die 
Vorsymmetrierung dieser einzelnen Parallelschaltungen erfolgt durch Messung und Selektion der Einzelkapazitäten.

Sowohl beim Lade- als auch beim Entladevorgang, werden die an den einzelnen Paketen anliegenden Spannungen überwacht und gesteuert, sodass beim Ladevorgang sichergestellt werden kann, dass neben dem Erreichen der maximalen Ladespannung für das Gesamtpaket auch die einzelnen Supercap-Pakete innerhalb ihrer Spezifikation von im Maximalfall $2,7 \mathrm{~V}$ bleiben. Bei einer vollständigen Entladung des Supercap-Paketes könnten negative Spannungen auftreten, die ebenfalls zu einer Schädigung oder Zerstörung von Einzelkondensatoren führen könnten. Deshalb wird auch hier eine Überwachung der einzelnen Spannungen vorgesehen und gegebenenfalls ein Energieaustausch realisiert.

Da die in den Supercaps enthaltene Leistung quadratisch mit der Spannung abnimmt, ist jedoch in der Praxis eine vollständige Entladung auch wegen des Wirkungsgrades der DC-DC-Wandler, der mit sinkender Spannung des Gesamtpaketes ab einem gewissen Grenzwert stark abnimmt, die Entladespannung auf etwa $30 \%$ der Maximalspannung begrenzt. Dieses führt zu einer möglichen Energieausbeute von etwa $90 \%$, was sich für den praktischen Einsatz als optimale Lösung gezeigt hat.

Das IFT geht davon aus, dass für kleine, mobile Fahrzeuge der Einsatz von Superkondensatoren wegen der hohen Energiedichte und der kurzen Ladezeiten in mittelbarer Zukunft zwingend notwendig sein wird. Über die Möglichkeit des zusätzlichen Einsatzes von Standardakkumulatoren zur Bereitstellung von großen Energiemengen werden derzeit noch unterschiedliche Untersuchungen getätigt. Das IFT geht derzeit auch davon aus, das für dieses Projekt der alleinige Einsatz von Superkondensatoren ausreichen wird, um die entsprechende Energiemenge zur Verfügung zu stellen und bei Einsatzszenarien von Fahrtentfernungen von bis zu etwa 200 Metern genügend Energie gespeichert werden kann, um auch bei Standzeiten von mehreren Tagen noch ein sicheres Anfahren der Fahrzeuge ermöglichen zu können.

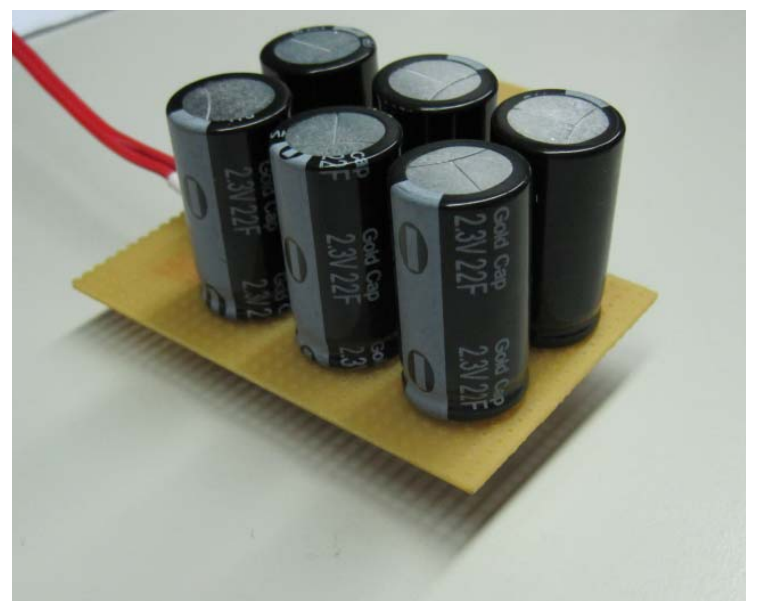

Abbildung 4: Erste Supercap-Pakete für Tests 
Zum Test der Symmetrierung der Supercap-Pakete und der Anwendung als Energiespeicher wurde ein Modell im Maßstab 1:10 aufgebaut, das heißt es wird ein Paket mit einem Zehntel der später im Prototypfahrzeug verwendeten Kapazität zum Test verwendet. Mit diesem Modell werden Lade- und Entladezyklen simuliert und auch Testfahrten mit den Antriebsmotoren auf dem Motorprüfstand des IFT gefahren.

\section{$4 \quad$ Antriebskomponenten}

Die Antriebskomponenten der beiden Fahrzeuge stellen einen wesentlichen Punkt bei den Kosten der Fahrzeuge dar. Klassischerweise werden Fahrzeugantriebe über einen einzelnen Fahrlenkantrieb oder einen Differentialantrieb gelöst. Beim Fahrlenkantrieb handelt es sich in der Intralogistik meist um ein einzelnes angetriebenes Rad, das mit einem zusätzlichen Lenkmotor die Fahrtrichtung und den Antrieb bestimmt. Ein typisches Anwendungsbeispiel hierfür sind Dreiradfahrzeuge. Beim Differentialantrieb verfügt das Fahrzeug über zwei fest montierte Fahrantriebe. Durch eine gegenläufige Ansteuerung der beiden Antriebe wird die Fahrtrichtung gewechselt, ähnlich wie bei Kettenfahrzeugen.

Zur Realisierung der geplanten Fahrzeuge scheidet der herkömmliche Fahrlenkantrieb aufgrund seiner aufwendigen Bauart und der damit verbundenen hohen Kosten aus. Ein reiner Differentialantrieb hingegen lässt sich leicht umsetzen und führt zu einer hohen Manövrierfähigkeit, zeigt jedoch ein relativ unruhiges Führungsverhalten und ruft ggf. einen starken Verschleiß hervor. Das KATE-Konzept sieht daher einen Lenkkopf mit zwei Differentialantrieben vor.

Der Lenkkopf bei diesem Konzept besteht aus einer in der Mitte drehbar gelagerten Achse deren beide Räder mit unabhängigen Antrieben versehen sind. Werden diese beiden Antriebe synchron angesteuert erfolgt keine Verdrehung der Achse und das Fahrzeug fährt gerade aus. Wird ein Antrieb schneller betrieben als der andere folgt eine Verdrehung der Achse und somit eine Kurvenfahrt des Fahrzeuges. Die Hinterachse des Fahrzeuges wird durch feststehende Rollen realisiert.

Beim Einsatz eines Lenkkopfes mit Differentialantrieb werden sehr hohe Anforderungen an das Ansteuer- und Regelverhalten der Antriebsmotoren gestellt. Um präzise Lenkbewegungen oder auch eine stabile Geradeausfahrt realisieren zu können müssen die Motoren bei sehr kleinen Drehzahlen und großen Momenten genau steuerbar sein.

Auf dem Markt sind zahlreiche unterschiedliche Motoren mit entsprechenden Getrieben verfügbar, die für den Einsatz in den Fahrzeugen geeignet sind. Dabei unterscheiden sich die verschiedenen Motorkonzepte (siehe Abbildung 5) durch systembedingte Vor- und Nachteile. 


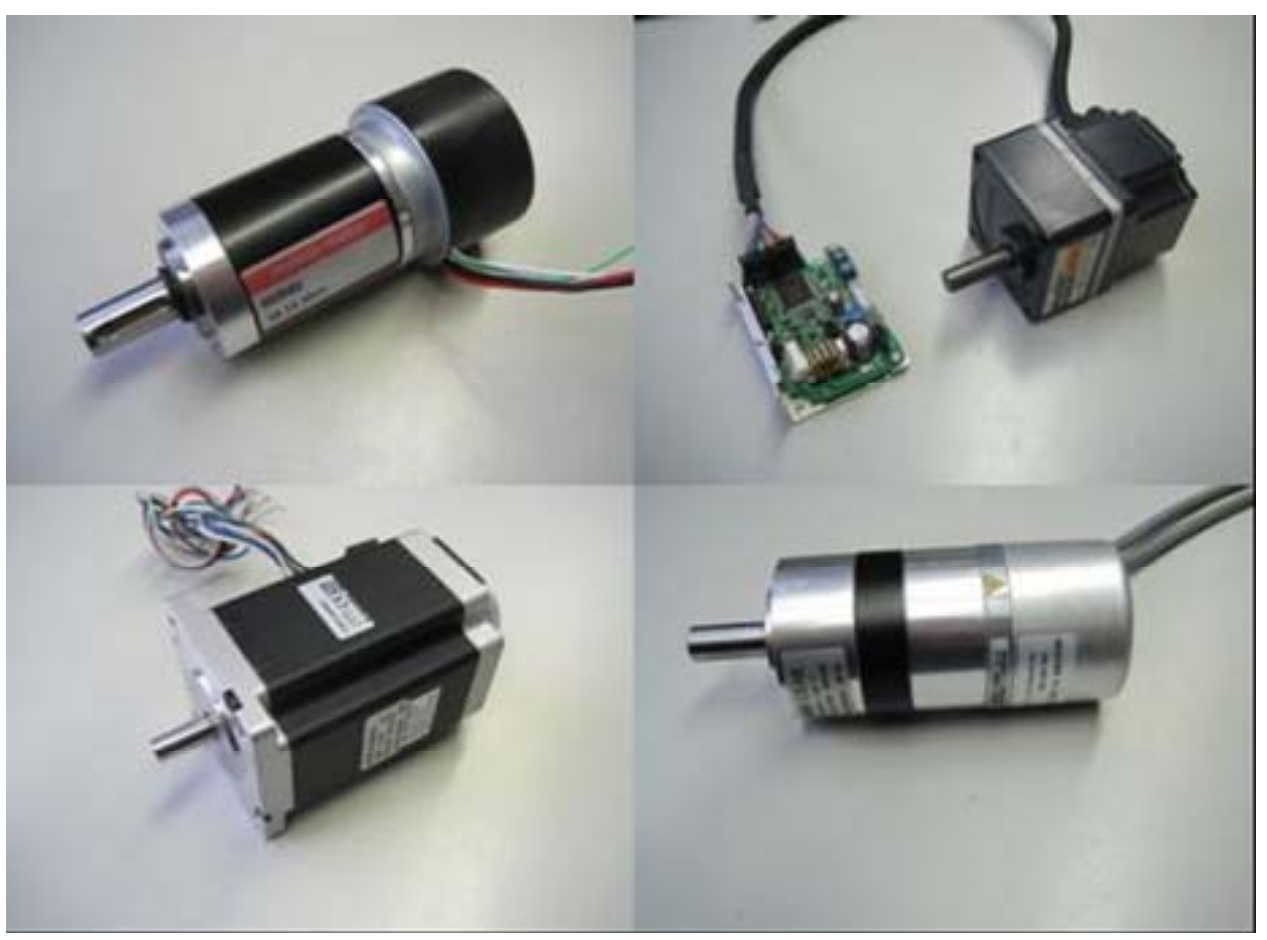

Abbildung 5: Verschiedene Motorvarianten

Viele der heute eingesetzten Kleinmotoren sind so genannte EC-Motoren. Bei diesen Motoren handelt es sich um bürstenlose permanent angeregte Synchronmotoren, die elektronisch kommutiert werden. Aufgrund dieser Bauweise erreichen EC-Motoren eine sehr hohe Dynamik. Da durch die elektronische Kommutierung keine Bürsten vorhanden sind, arbeitet der Motor bis auf die Lager verschleißfrei. An die Motoren werden je nach benötigtem Drehmoment oder Drehzahl Planeten- oder Stirnradgetriebe angeflanscht.

Alternativ sind Schrittmotoren als Antriebsmotoren für die Fahrzeuge denkbar. Schrittmotoren sind im Prinzip hochpolige Synchronmotoren, bei denen sich der so genannte Rotor durch ein gesteuertes schrittweise rotierendes elektromagnetisches Feld der Statorspulen um einen minimalen Winkel dreht. Schrittmotoren bieten den Vorteil sehr exakte Bewegungen ausführen zu können und dabei bezogen auf Ihre Baugroße ein hohes Moment aufbringen können. Dadurch können beim Einsatz in den Fahrzeugen die Getriebe entfallen. Allerdings ist der Wirkungsgrad der Schrittmotoren stark von der Betriebsart abhängig und in der Regel deutlich schlechter als z.B. bei EC-Motoren.

$\mathrm{Da}$ an die Antriebsmotoren sehr hohe Anforderungen bezüglich der Regelbarkeit gestellt werden und die Angaben der Motorenhersteller nur sehr wenige Aussagen über die tatsächliche Leistungsdaten geben wurden im Rahmen des Projektes verschiedene Antriebsmotoren auf einen Prüfstand getestet. Auf diesem Prüfstand konnte darüber hinaus der Wirkungsgrad und Stromverbrauch (Spitzenstrom) der verschiedenen Motoren ermittelt werden. Dies sind wesentliche Grundlagen für die Auslegung des Energiespeichers. 
Klassische Motorenprüfstände werden in der Regel mit einem Prüfmotor der über eine Drehzahl-Drehmomentmesswelle eine Last (z.B. Bremse oder Generator) antreibt realisiert. Dieses Konzept lässt sich bei derart kleinen Antrieben jedoch nur mit sehr hohem Aufwand realisieren. Aus diesem Grund wurde für den im Rahmen des Projektes realisierten Prüfstand ein Konzept gewählt bei dem der Motor über eine Gelenkwelle direkt eine Seiltrommel (Abbildung 6) antreibt. Auf die Seiltrommel wird ein dünnes Seil gewickelt, an dessen Ende verschiedene Gewichte angebracht werden können.
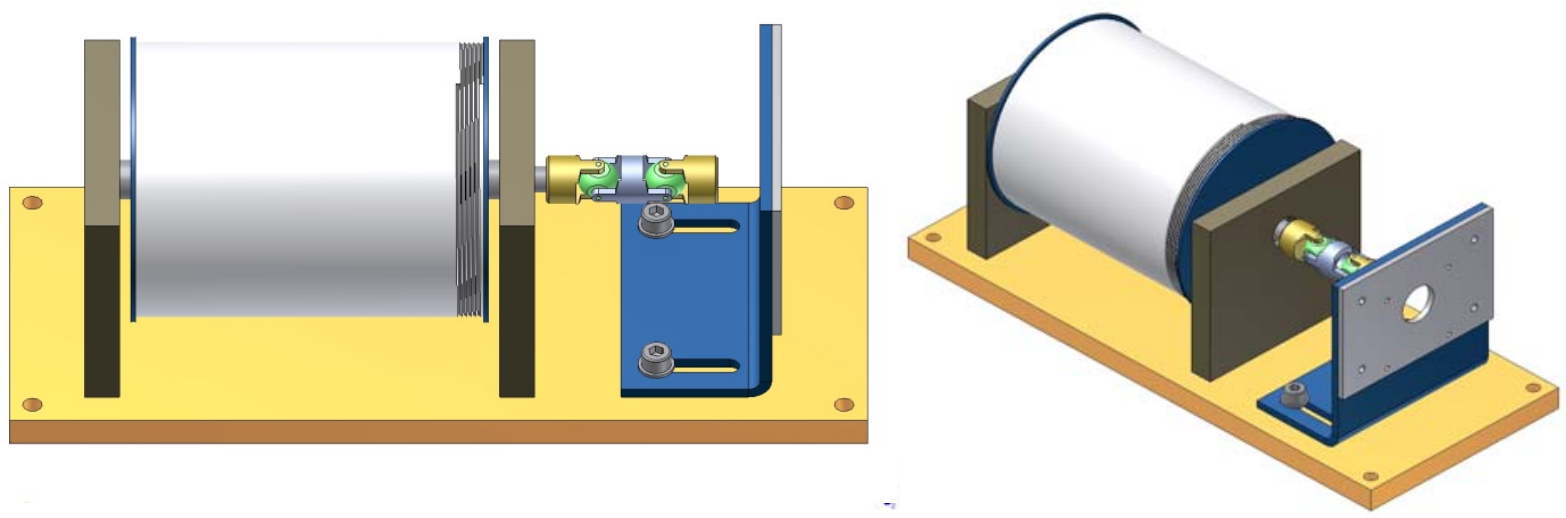

Abbildung 6: Motorenprüfstand IFT

Dieses Konzept bietet den Vorteil, dass das Antriebsmoment direkt durch das angehängte Gewicht bestimmt wird und somit nicht an der sich drehenden Welle gemessen werden muss. Darüber hinaus bleibt das Moment während des Prüflaufes (bei Konstantfahrt) nahezu konstant. Während der Prüfläufe wurden alle relevanten Messgrößen mittels einer Messkarte durch einen Mess-PC erfasst. Über die Ausgänge der Messkarte erfolgte die Ansteuerung der Motoren. So konnten vordefinierte Prüfabläufe exakt identisch für alle Motoren durchgeführt werden. Während der Versuche wurden fortlaufend die Versorgungsspannung und die Stromaufnahme gemessen, so dass die aufgenommene elektrische Leistung ermittelt werden konnte. Die Messung der Drehzahl des Motors erfolgte über die Messung des zurückgelegten Weges des Gewichtes. Hierzu wurde ein LaserLängenmesssystem verwendet. Darüber hinaus wurden je nach Motor die Encodersignale aufgezeichnet, um deren Verwendbarkeit für den späteren Fahrzeugrechner beurteilen zu können.

\section{$5 \quad$ Bewegungsmöglichkeiten der Transporteinheiten}

Aufgrund des komplett symmetrischen Aufbaus gibt es bei den Transportkufen keine feste Vorder- und Hinterachse [DE07]. Je nach Fahrtrichtung kann eine Achse einer Transportkufe vor der zweiten Achse liegen, oder beide Achsen liegen auf gleicher Höhe (z.B. bei Fahrt quer zur Längsrichtung der Transportkufen). 


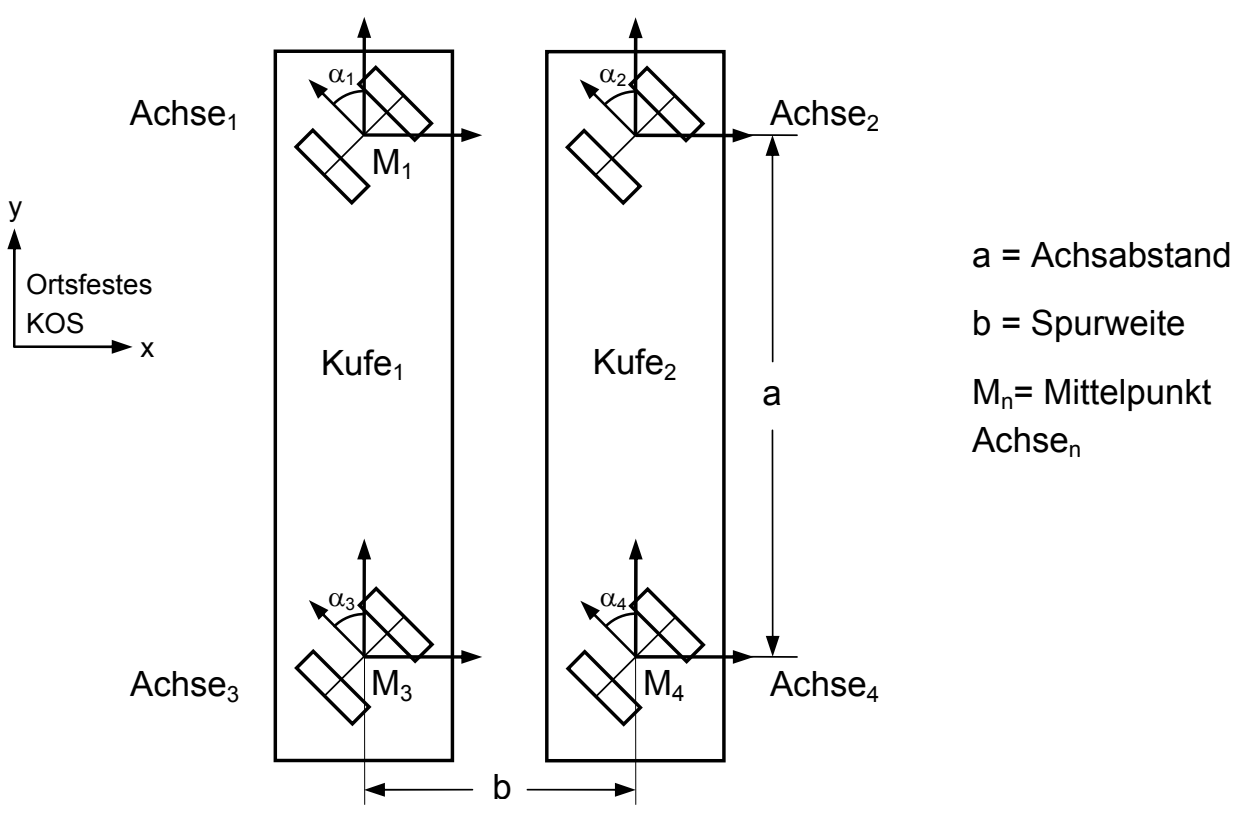

Abbildung 7: Koordinatensysteme, Winkel

Bei stationärer Kurvenfahrt (nur Achse 1 und Achse 2 gelenkt) muss (für geringe Geschwindigkeiten) gelten $\tan \alpha_{1}=a / c$ und $\tan \alpha_{2}=a /(b+c)$. Die Lenkung der Achsen entspricht einer Ackermann-Lenkung. Dies ist über die Steuerung der Achsen 1 und 2 sicherzustellen.

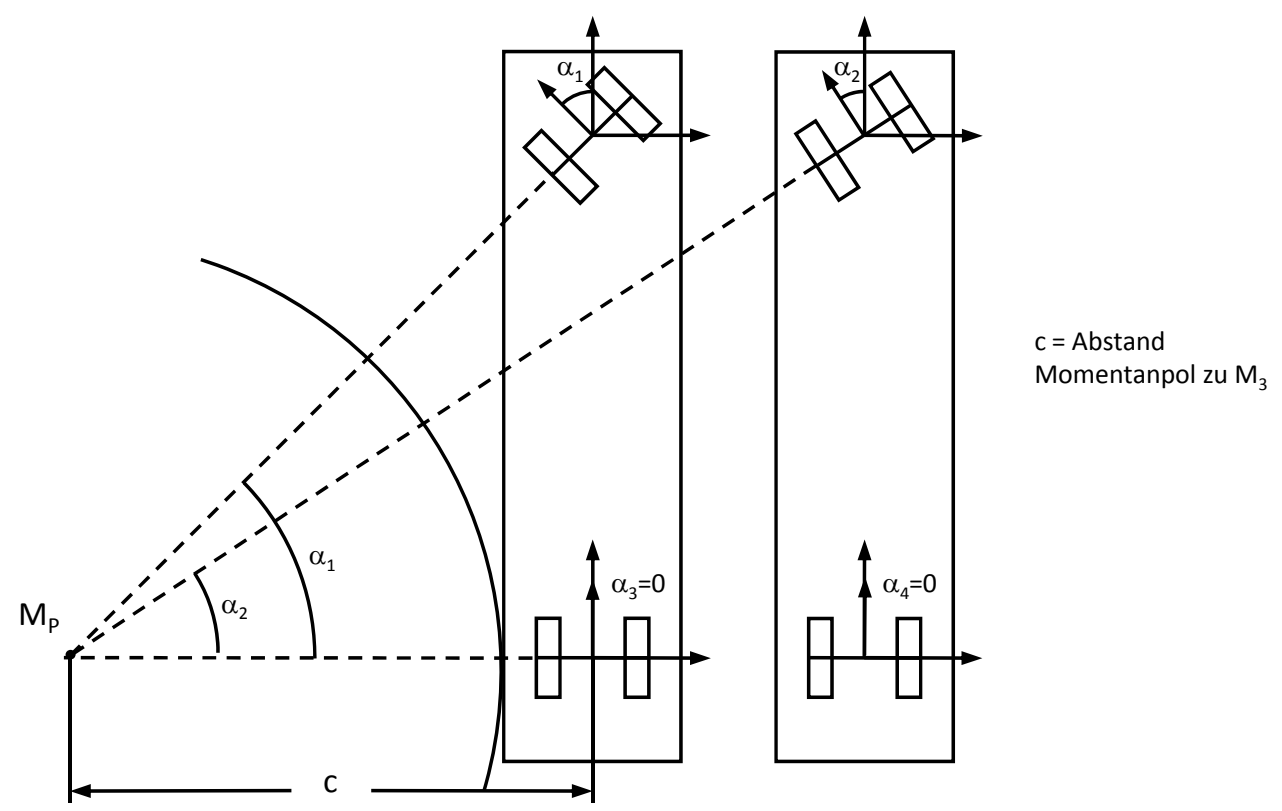

Abbildung 8: Lage des Momentanpols bei doppelter Drehschemellenkung

Werden alle Achsen gelenkt und der Momentanpol liegt mittig in Fahrzeuglängsrichtung so ergibt sich $\alpha_{3}=-\alpha_{1}$ und $\alpha_{4}=\alpha_{2}$, sowie $\alpha_{1}=\arctan (a / 2 / c)$. Der Abstand c verringert sich durch Lenken aller Achsen $\left(\alpha_{1}=-\alpha_{3}\right)$ im Vergleich zur Variante mit nur einer Lenkachse (bei betragsmäßig gleichen Lenkwinkeln $\alpha_{1}$ ) deutlich, siehe Abbildung 8 und Abbildung 9. Daraus ergibt sich ein deutlich geringerer innerer Wenderadius. Bei dem KATE-Konzept gilt jeweils $\alpha_{3}=\alpha_{4}=0^{\circ}$. 

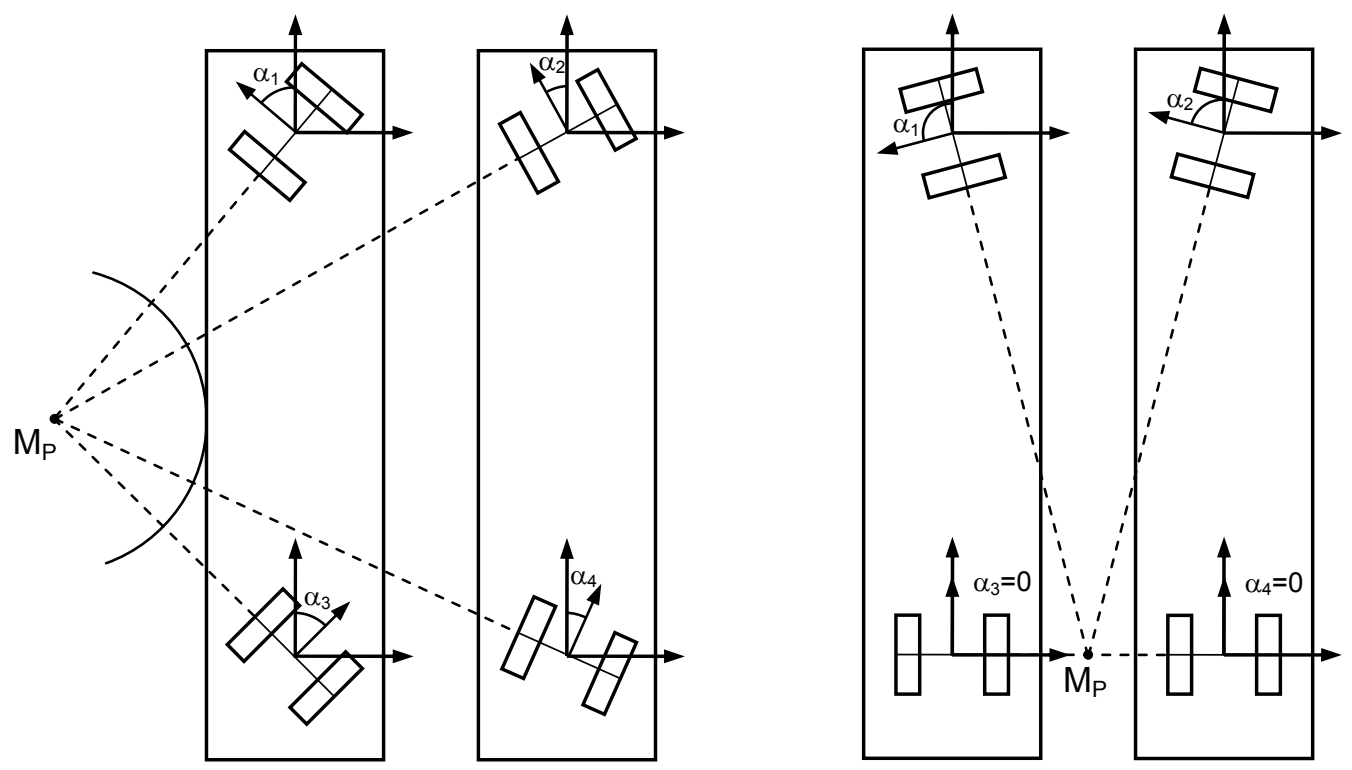

Abbildung 9: Zwei mögliche Lagen des Momentanpols bei Allradlenkung

Werden die Lenkwinkel an den Achsen 1 und 2 so eingestellt, dass sich die Strahllinien zwischen $\mathrm{M}_{3}$ und $\mathrm{M}_{4}$ treffen und die Achsen 3 und 4 werden nicht gelenkt $\left(\alpha_{3}=\alpha_{4}=0\right)$ so verhalten sich die Transportkufen wie ein Dreiradstapler. Durch entsprechende Wahl der Lenkwinkel der einzelnen Achsen können die unterschiedlichsten Lenk- und Bewegungsmöglichkeiten in alle 2 Raumrichtungen durchgeführt werden.

\subsection{Lenk-Möglichkeiten zum Durchfahren einer Kurve}

Soll eine Kurve kontinuierlich durchfahren werden, so ergeben sich aufgrund der omnidirektionalen Bewegungsmöglichkeiten mehrere unterschiedliche Varianten. In Abbildung 10 sind die unterschiedlichen Möglichkeiten eine Kurve zu durchfahren dargestellt. Zunächst können nur die Achsen 1 und 2 gelenkt werden. Stimmt die Fahrtrichtung mit der positiven y-Achse überein, so handelt es sich um die „Vorderachsen“. Die Achsen 3 und 4 werden nicht gelenkt und die Transportkufen lassen sich wie ein Auto (jedoch mit größerem Einschlagwinkel) um die Kurve steuern. Alternativ könnten nur die Achsen 3 und 4 gelenkt werden, wodurch sich die Transportkufen wie ein Vierrad- oder ein Dreirad-Stapler lenken lassen. Werden alle vier Achsen gelenkt, so kann die Transportvorrichtung wie ein Vier-Wege-Stapler manövriert werden. 

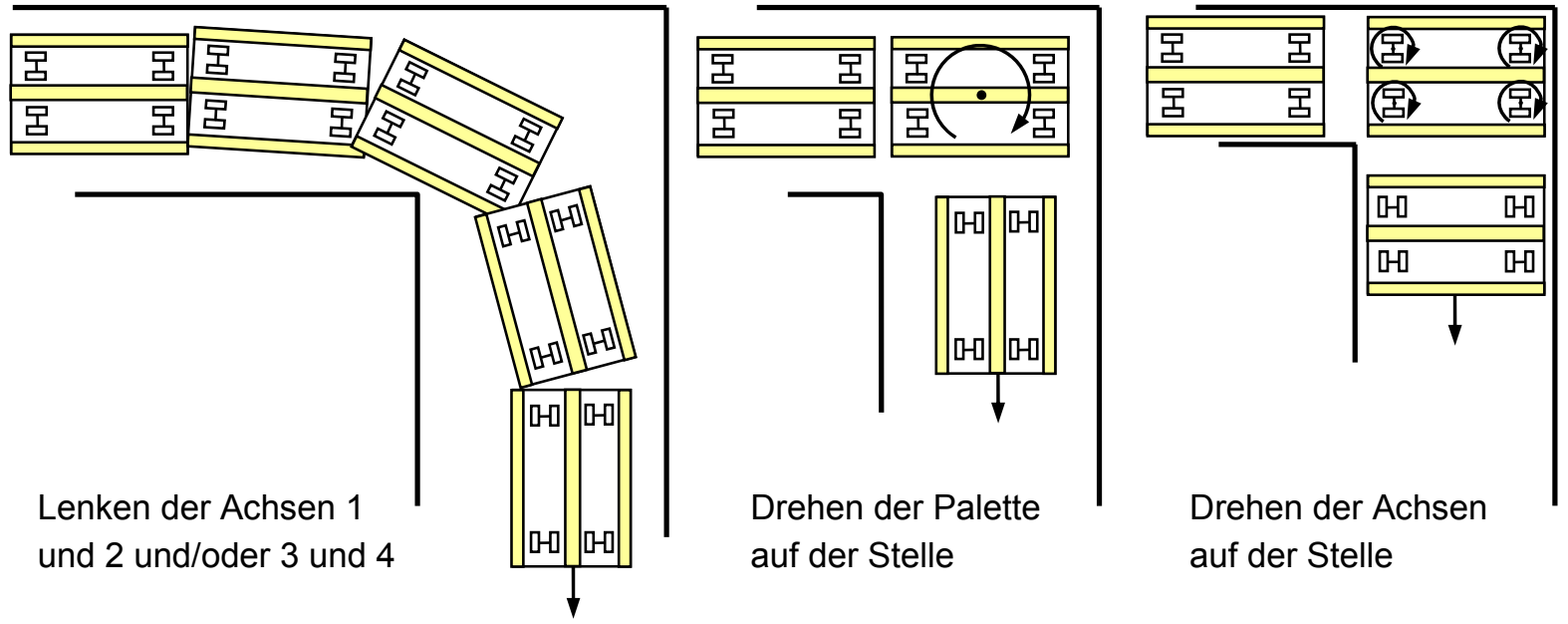

auf der Stelle

Abbildung 10: Unterschiedliche Möglichkeiten, Kurven zu durchfahren

Neben dem kontinuierlichen Durchfahren einer Kurve kann auch mit einem kurzen Stopp die Palette auf der Stelle gedreht werden $\left(\alpha_{1}=-\alpha_{2}\right.$ und $\left.\alpha_{3}=-\alpha_{4}\right)$. Um auf minimalem Raum die Fahrtrichtung der Palette zu ändern ist es ebenso möglich nur die Achsen der Transportkufen auf der Stelle zu drehen. Die Palette wird nicht mitgedreht, sondern bewegt sich nun in x- statt in y- Richtung, wobei die Orientierung der langen und kurzen Seite sich nicht ändert, siehe Abbildung 10.

\subsection{Weitere Verfahrmöglichkeiten}

Eine weitere Verfahrmöglichkeit die sich durch die Allradlenkung bietet ist der Hundegang. Die Besonderheit des Hundegangs ist, dass die Paletten gleichzeitig in $x$ - und $y$-Richtung bewegt werden können. Dies bietet insbesondere beim Positionieren von Paletten in beengten Platzverhältnissen (z.B. auf einem LKW) besondere Vorteile (Abbildung 11). Bei einem Handgabelhubwagen kann z.B. aufgrund der Kinematik der Momentanpol der Drehbewegung nur auf der Linie der Achsen $\mathrm{H}_{2}$ und $\mathrm{H}_{3}$ liegen. Somit ist es unmöglicht eine Palette optimal (in $\mathrm{x}$ - und $\mathrm{y}$ Richtung $0 \mathrm{~mm}$ Abstand zur Wand) in einer Ecke abzustellen, ohne mit der Ecke der Palette unsanft gegen die Wand zu fahren bzw. davor bereits mit Abstand $\Delta x=0$ mit der Palette an der Wand entlang zu fahren oder die Palette nach dem Abstellen noch translatorisch zu verschieben. Mit dem Hundegang ist ein optimales Positionieren im Eck möglich. 


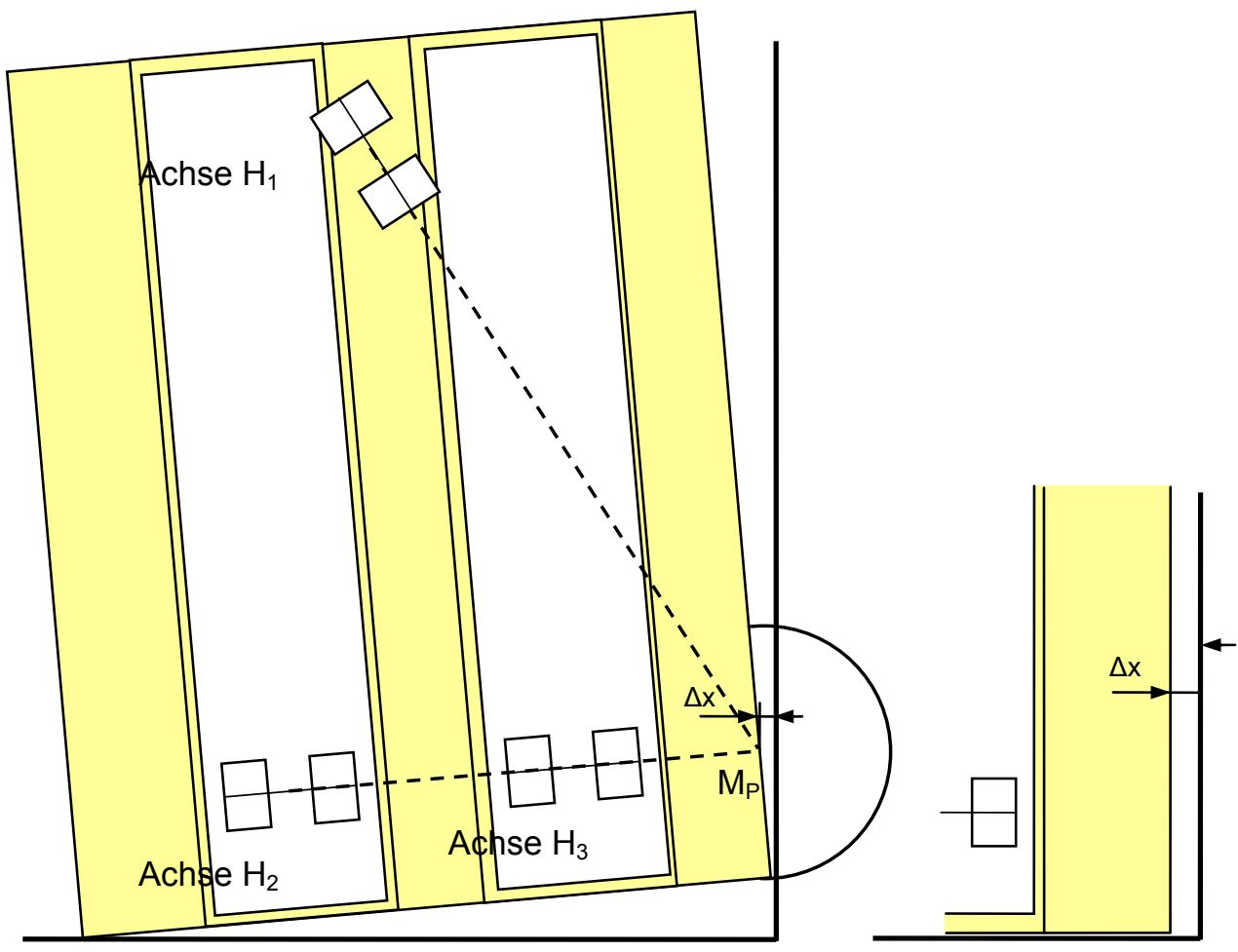

Abbildung 11: Problematik beim genauen Positionieren einer Palette

\section{Zusammenfassung und Ausblick}

Im Rahmen der noch am IFT laufenden Forschungsprojekte wurden erste Prototypenfahrzeuge aufgebaut mit denen bereits Testfahrten durchgeführt werden konnten. Bei den Untersuchungen mit diesen Prototypen liegt das Hauptaugenmerk auf dem Test der Mechanik und der Energiespeicherung. Dabei wird die Fahrzeugsteuerung erprobt und optimiert. Parallel zu diesen Untersuchungen wird an einem neuen Navigationssystem für die Fahrzeuge gearbeitet, welches neben weiterer Sensortechnik z.B. zur Kollisionserkennung in die Prototypenfahrzeuge integriert und getestet werden soll. Hierdurch sollen letztlich auch Verbundfahrten mehrerer Fahrzeuge ermöglicht werden.

\section{Literatur}

[DE07] DE102007046868 A1: Transportvorrichtung für Ladungsträger und Verfahren zu deren Steuerung

[Goe11] Götting KG: Optische Spurführung. www.goetting.de: Februar 2011

[Max11] Maxwell Technologies, Inc: www.maxwell.com: Februar 2011 